\title{
Evidence of a Distinct Behavioral Phenotype in Young Boys with Fragile X Syndrome and Autism
}

\author{
Jason J. Wolff, Ph.D., James W. Bodfish, Ph.D., Heather C. Hazlett, Ph.D., Amy A. \\ Lightbody, Ph.D., Allan L. Reiss, M.D., and Joseph Piven, M.D. \\ Drs. Wolff, Bodfish, Hazlett, and Piven are with the Carolina Institute for Developmental \\ Disabilities and the University of North Carolina at Chapel Hill. Drs. Lightbody and Reiss are with \\ the Center for Interdisciplinary Brain Science Research and Stanford University
}

\begin{abstract}
Objective-How does the behavioral expression of autism in fragile X syndrome (FXS+Aut) compare to idiopathic autism (iAut)? While social impairments and restricted, repetitive behaviors (RRBs) are common to both variants of autism, closer examination of these symptom domains may reveal meaningful similarities and differences. To this end, we profiled the specific behaviors comprising the social and repetitive behavioral domains in young children with FXS+Aut and iAut.

Method-Twenty-three males ages 3-5 years with FXS + Aut were age-matched with a group of 38 boys with iAut. Repetitive behavior was assessed using the RBS-R. Social behavior was evaluated using Autism Diagnostic Observation Schedule (ADOS) social item severity scores.

Results-Rates of stereotypy, self-injury, and sameness behaviors did not differ between groups, whereas compulsive and ritual behavior scores were significantly lower for individuals with FXS + Aut compared to iAut. Those with FXS + Aut scored significantly lower (less severe) than the iAut group on five ADOS measures of social behavior: Gaze Integration, Quality of Social Overtures, Social Smile, Facial Expressions, and Response to Joint Attention.
\end{abstract}

Conclusions-The behavioral phenotype of FXS + Aut and iAut are most similar with respect to lower-order (motoric) RRBs and social approach, but differ in more complex forms of RRB and some social response behaviors. These findings highlight the phenotypic heterogeneity of autism overall and its unique presentation in an etiologically distinct condition.

\section{Keywords}

fragile X syndrome; autism; repetitive behavior; behavioral phenotype

\footnotetext{
(C) 2012 American Academy of Child \& Adolescent Psychiatry. Published by Elsevier Inc. All rights reserved.

Correspondence to Jason Wolff, Ph.D., Carolina Institute for Developmental Disabilities, University of North Carolina at Chapel Hill, CB\# 3367, Chapel Hill, NC 27599-3367; jason.wolff@ cidd.unc.edu. Drs. Reiss and Piven contributed equally to this research.

Publisher's Disclaimer: This is a PDF file of an unedited manuscript that has been accepted for publication. As a service to our customers we are providing this early version of the manuscript. The manuscript will undergo copyediting, typesetting, and review of the resulting proof before it is published in its final citable form. Please note that during the production process errors may be discovered which could affect the content, and all legal disclaimers that apply to the journal pertain.

Dr. Wolff served as the statistical expert for this research.

Disclosure: Dr. Reiss has served as a consultant for Novartis. Drs. Wolff, Bodfish, Hazlett, Lightbody, and Piven report no biomedical financial interests or potential conflicts of interest.
} 
Fragile X syndrome (FXS) is the most common known inherited cause of intellectual disability (ID). ${ }^{1}$ The behavioral phenotype of FXS includes a number of classically "autistic" features, including motor stereotypies and self-injurious behavior (SIB), perseverative behavior, social avoidance, poor eye contact, and odd or delayed speech. ${ }^{2-7}$ Because of phenotypic overlap with idiopathic autism (iAut), FXS is increasingly considered a subtype of autism or itself one of the "autisms". 8,9 Approximately one third of boys with FXS meet $D S M-I V$ criteria for autism, with nearly a third more meeting criteria for a pervasive developmental disorder. 5,7

If the FXS phenotype includes many classically autistic features, what differentiates children with FXS who receive a diagnosis of autism? It has been suggested that excess repetitive behavior may account for many of the autistic symptoms associated with FXS. ${ }^{10-12}$ For example, stereotypical manipulation of objects differentiates children with FXS + Aut from those without an autism diagnosis. ${ }^{13,14}$ This is notable given that repetitive object manipulation is among the earliest distinct markers of iAut. ${ }^{15}$ However, other forms of repetitive behavior, such as compulsivity or SIB, are elevated in children with FXS regardless of the presence of autism. ${ }^{16,17}$ Separate lines of research have identified key differences in social behaviors between those with FXS with and without autism. ${ }^{6,7}$ Social avoidance and failure to recognize social cues may drive a diagnosis of autism in FXS, though impairments in this domain are expressed on a continuum in FXS regardless of comorbid condition. ${ }^{18,19}$ Because social avoidance or anxiety are common to children with FXS generally, it may not reliably distinguish those with and without autism. ${ }^{20}$ Minor degrees of difference in social performance may determine whether a given individual falls just above or below the level of clinical significance on autism assessments. ${ }^{6,20,21}$ As of yet, the precise constellation of repetitive and social behaviors constituting autism in FXS remains unclear.

Studies comparing individuals with FXS with and without autism only partially informs the phenotype of FXS+Aut. By definition, autistic behaviors are more severe in FXS+Aut relative to FXS without autism. There are comparatively fewer direct comparisons between FXS + Aut and iAut. Bailey et al. ${ }^{22}$ found that FXS + Aut is characterized by a similar but generally milder profile of autistic symptoms compared to iAut. Others have found that children with FXS + Aut closely resemble those with iAut on total and symptom domain scores taken from both the Autism Diagnostic Interview (ADI-R) or Autism Diagnostic Observation Schedule (ADOS). ${ }^{7,} 23$ It is worth noting that most group comparisons utilize summary-level diagnostic measures. This approach generally lacks the precision necessary to identify specific behavioral differences. Studies comparing discrete social behaviors between groups have found that those with FXS + Aut show significantly less impairment on measures of social smiling, shared enjoyment, and amount and quality of social interactions. ${ }^{6,}{ }^{11} \mathrm{Kau}$ et al. ${ }^{11}$ concluded that given their intermediate profile of social impairments, children with FXS+Aut likely receive an autism diagnosis due to high rates of repetitive behavior and limited communication ability. This view is supported in part by recent findings showing significantly higher rates of repetitive behavior in adolescents and adults with FXS+Aut compared to individuals with iAut. ${ }^{24}$

Autism is highly heterogeneous, but its association with FXS affords the opportunity to refine a behavioral phenotype against a stable genetic background. A fine-grained examination of the FXS+Aut phenotype would clarify this specific variant of autism and inform studies seeking to establish gene-brain-behavior relationships. ${ }^{25,}{ }^{26}$ In the present study, we aimed to: 1) Characterize constituent features of repetitive and social behaviors in young males with FXS + Aut, and 2) Compare and contrast those patterns of behavior with a comparison group of young males with iAut. Because the expression of autistic behaviors 
are known to change over time, the present study focused on a single age cohort (3-5 year olds) to control for the effects of age on behavior. ${ }^{14,27}$

\section{Method}

\section{Participants}

Participants were part of a collaborative imaging study of FXS. ${ }^{28,}{ }^{29}$ Children with FXS were recruited through regional and national FXS organizations and research registries maintained by either Stanford University or the University of North Carolina (UNC). A comparison group of children with iAut were recruited through UNC's Division Treatment and Education of Autistic and Related Communication-Handicapped CHildren (TEACCH) and Stanford area clinics. Exclusion criteria for the parent study included evidence of tuberous sclerosis (TS), history of central nervous system (CNS) injury (e.g., cerebral palsy, significant pregnancy complications or perinatal/postnatal trauma, drug exposure), prematurity ( $<34$ weeks), low birth weight $(<2000 \mathrm{~g})$, seizures, and significant motor or sensory impairments. For the present study sample, children ages 3 to 5 with: a) complete Repetitive Behavior Scales (RBS-R, ${ }^{30}$ ), and b) an Autism Diagnostic Observation Schedule $\left(\right.$ ADOS- $\mathrm{G}^{31}$ ) classification of autism and Autism Diagnostic Interview (ADI- $\mathrm{R}^{32}$ ) scores consistent with this classification were included, yielding a total cross-sectional sample of 23 males with FXS + Aut (mean age 4, SD=.8) and 38 with iAut (mean age 4.2, $S D=1$ ). Full mutation FXS ( $>200$ CGG repeats) was confirmed with the standard Southern Blot technique and testing for Fragile X protein (FMRP) expression by calculating the percentage of peripheral lymphocytes containing FMRP. ${ }^{33}$ Participants in the iAut group were excluded for evidence of FXS. Participants meeting ADOS criteria for "autism spectrum" were not included in either the iAut or FXS + Aut groups. Study approval was acquired from University of North Carolina and Stanford University Institutional Review Boards and written informed consent obtained from parents or custodial guardians for each participant.

\section{Measures}

The RBS-R is a 43 item parent-rated measure of discrete types of repetitive behavior and yields both total and subscale scores. ${ }^{30}$ The RBS-R is comprised of six subscales: stereotyped behavior, self-injurious behavior, compulsive behavior, ritualistic behavior, sameness behavior, and restricted behavior. The RBS-R has been independently validated for use among young children with autism. ${ }^{34}$

The ADOS is a standardized observational measure of behaviors associated with autism. It includes a variety of semi-structured activities and presses intended to assess communicative, social, and play behavior. ${ }^{31}$ Individual items from the social domain of the ADOS were used to compare performance on specific social behaviors in a manner similar to recent work by Hall et al. ${ }^{6}$ To examine whether social avoidance or anxiety rather than social indifference drives social deficits in FXS + Aut, we were specifically interested in those social behaviors representing social initiation versus social responsiveness. ${ }^{11,18}$ The 12 items comprising the ADOS-G module 1 social domain were rationally derived into 3 categories prior to analysis based on item-level behavioral coding descriptions and experience administering the measure to young children. Items were classified as measuring: 1) primarily social initiation, 2) primarily social response, and 3) unable to classify. Those behaviors determined to primarily measure social initiation were: Integration of Gaze, Requesting, Giving, Showing, Initiation of Joint Attention (IJA), and Quality of Social Overtures. Those behaviors determined to primarily measure social response were: Response to Name, Responsive Social Smile, Facial Expressions (socially directed), Shared Enjoyment, and Response to Joint Attention (RJA). One item, Unusual Eye Contact, could not be classified as either primarily a measure of social initiation or social response and was 
not included in our analyses. ADOS item scores of ' 2 ' and ' 3 ' were collapsed into a single score, consistent with ADOS severity scoring convention. ${ }^{35}$ Though all participants had total ADOS algorithm scores, 5 children in the iAut group were not included in social domain analyses ( 4 with missing item level data and 1 with module 2 scores). The Mullen Scales of Early Learning, a standardized measure of cognitive and motor development for use in early childhood, was administered to all participants. ${ }^{36}$ Mullen Early Learning Composite (ELC) standard scores and derived ratio IQ scores were used to characterize general intellectual ability. All behavioral assessments were administered by trained clinicians at either Stanford University or UNC. Inter-rater reliability was initially established and regularly monitored between raters and sites.

\section{Analyses}

Statistical tests were performed using SPSS 18 (SPSS Inc., Chicago IL). For the purposes of this study, RBS-R subscale scores were generated and compared between groups (FXS + Aut and iAut). Because each subscale contains a different number of items, comparisons cannot be made across subscales, but may be made between groups on the same given domain of repetitive behavior. ADOS social item mean scores were generated for both groups and sorted into domains of social initiation or social response. Mean RBS-R subscale scores and ADOS social initiation and social response items were compared using type-III sum of squares multiple-analysis of covariance, with group status fit as the dependent variable and ratio IQ scores (derived from Mullen age equivalent scores) as a covariate.

As a follow-up analysis, a subset of the iAut group was matched to the FXS + Aut group using SPSS on the basis of ratio IQ scores. Those RBS-R or ADOS items that differed between groups in the original sample were compared between developmentally matched groups through Bonferroni corrected multiple-analysis of variance.

\section{Results}

The mean age of administration for the RBS-R was $4.0(S D=.8)$ for the FXS + Aut group and $4.2(S D=1)$ for iAut group. There were no significant group differences by age of RBS$\mathrm{R}$ administration, $t(59)=1.5, p=.16$. The mean age of ADOS and Mullen assessments for the FXS + Aut group was $3.8(S D=.7)$ and $3.6(S D=1)$ for the iAut group. Groups did not differ significantly on age of ADOS or Mullen assessments, $t(54)=1.1, p=.27$. As expected, mean Mullen ELC scores differed significantly between FXS + Aut ( $M=49.8$, $S D=2.6)$ and iAut $(M=62, S D=20.1), t(59)=2.9, p=.005$. Because Mullen ELC standard scores for $78 \%$ of boys with FXS+Aut and $56 \%$ of boys with iAut were at the floor of the measure, ratio IQ scores were computed based on Mullen age equivalent scores ([mental age (MA)/chronological age (CA) $\left.{ }^{*} 100\right)$. Mean ratio IQ for boys with FXS+Aut was $30.2(S D=16.3$; range: $8.9-60.8)$, and $38.4(S D=19.2$; range: $14.2-92)$ for boys with iAut. Ratio IQ scores were fit as a covariate in subsequent models.

\section{Repetitive and Social Behavior, FXS + Aut vs. iAut}

Omnibus multivariate analysis of covariance (MANCOVA) results for RBS-R subscale scores were at the level of significance (Wilks' lambda $=.8, F^{6,53}=2.3, p=.05$ ). Among RBS-R subscales, the iAut group scored significantly higher (more severe) than FXS + Aut for compulsive and ritual behavior. Stereotypy, self injurious behavior (SIB), sameness, and restricted behavior did not differ significantly between groups. Group descriptive and MANCOVA test statistics for RBS-R subscale items are presented in Table 1. Mean subscale scores with $95 \%$ confidence intervals are presented for FXS + Aut and iAut groups in Figure 1. 
Omnibus test results for social initiation cluster was below the level of significance (Wilk's lambda $=.71, F^{5,50}=3.2 p=.01$ ). For comparisons of ADOS social items constituting social initiation, the FXS + Aut group scored significantly lower (less severe) on measures of Gaze Integration and Quality of Social Interactions. Groups did not differ significantly on measures of Requesting, Giving, Showing, and IJA. For the social response domain, omnibus test results indicated a significant difference between groups (Wilks' lambda $=.53$, $\left.F^{5,50}=8.8, p<.001\right)$. For ADOS social items classified as primarily social response, the FXS + Aut group scored significantly lower (less severe) on measures of Social Smile, Facial Expressions, and RJA. Group descriptive and MANCOVA test statistics for both social initiation and social response domains are presented in Table 2. Mean social initiation and social response scores for groups with 95\% confidence intervals are presented in Figures 2 and 3 , respectively.

\section{Matched-Sample Follow-up}

In order to more directly control for the potential effects of intellectual ability on repetitive and social behaviors, a subset of the iAut was matched to the FXS + Aut sample on the basis of ratio IQ scores computed from the Mullen, yielding 23 boys in each of the FXS + Aut and iAut groups. The mean IQ score for this matched iAut sample was $32.7(S D=15)$. This matched sample did not differ from the FXS + Aut group, $t(44)=.07, p=.95$ on general cognitive development as measured by the Mullen. The matched groups did not differ on age at administration of either RBS-R $[t(44)=1.2, p=.25]$ or ADOS [t(44) $=-.9, p=.36]$ measures. RBS-R subscales and ADOS social items for which groups significantly differed in the original analysis were included in the matched-sample follow-up. FXS + Aut and iAut groups were compared on RBS-R compulsive and ritual behavior subscales; ADOS social initiation domain items Gaze Integration and Quality of Social Overtures; ADOS social response domain items Social Smile, Facial Expressions, and RJA. These analyses were Bonferroni adjusted for multiple comparisons.

Compulsive behavior scores for FXS + Aut $(M=2.2, S D=1.8)$ were significantly lower than those for iAut $(M=5.9, S D=3.8), F^{1,45}=18.7, p<.01$. Ritual behavior scores for FXS + Aut $(M=2, S D=2.3)$ were significantly lower than those for iAut $(M=4.5, S D=$ $2.9), F^{1,45}=10.8, p=.01$. For ADOS social initiation domain items, scores for Quality of Social Overtures score were significantly lower (less impaired) for FXS + Aut ( $M=1.4, S D$ $=.5)$ than iAut $(M=1.8, S D=.4), F^{1,45}=8.3, p=.04$. Gaze Integration scores did not differ significantly between FXS + Aut $(M=1.4, S D=.7)$ and iAut $(M=1.9, S D=.3)$, $F^{1,45}=6.3, p=.11$. For ADOS social response items, FXS + Aut scores for Social Smile were significantly lower for FXS + Aut $(M=.9, S D=.9)$ than iAut $(M=1.9, S D=.3)$, $F^{1,45}=20.6, p<.01$. Scores for Facial Expressions were significantly lower for FXS + Aut $(\mathrm{M}=1.1, \mathrm{SD}=.5)$ than iAut $(\mathrm{M}=1.7, \mathrm{SD}=.4), F^{1,45}=17, p<.01$. Scores for RJA were significantly lower for FXS + Aut $(M=.7, S D=.9)$ and iAut $(M=1.4, S D=.8), F^{1,45}=$ $8.2, p=.049$.

\section{Comparison to FXS without Autism}

To provide additional context to the profile of behaviors observed in boys with FXS+Aut, social and repetitive behavior data were compiled for 27 boys with FXS without (w/o) Aut. This group included boys ages 3-5 from the parent study who did not meet cutoffs for autistic disorder. The mean age for this group was 3.6 $(S D=.8)$ for ADOS and Mullen assessment and $4.2(S D=1)$ for the RBS-R. The mean Mullen ELC for this group was 57.9 $(S D=13.3)$. There were no significant differences in age or ELC scores between FXS w/o Aut, and either FXS+Aut or iAut. Radar graphs were generated for RBS-R and ADOS social domain data and are presented in Figure 4. These graphs illustrate patterns of behavior at the item level for boys in three groups: iAut, FXS+Aut, and FXS w/o Aut. 


\section{Discussion}

This study examined patterns of repetitive and social behavior in two groups of young boys with autistic disorder - those with idiopathic autism (i.e., without FXS) and those with FXS. Though we found similarities in some aspects of repetitive and social behaviors, a number of key differences were identified. With regard to repetitive behavior, we observed the greatest degree of symptom overlap in repetitive motor behaviors (stereotypy, self-injury), but significantly less compulsive or ritual behavior in boys with FXS+Aut. This distinction is important given that repetitive motor behaviors are linked to developmental disability in general and are not specific to autism. ${ }^{37,38}$ While our division of ADOS items by initiation versus response did not fully delineate the social domain for boys with FXS+Aut, omnibus results for these divisions do provide partial support for this approach. Boys with FXS+Aut were characterized by significantly less impairment on measures of social response including 'Response to Joint Attention', 'Responsive Social Smile', and 'Facial Expressions', with effect sizes for items 'Responsive Social Smile' and 'Facial Expressions' particularly robust. These social behaviors appear relatively intact among boys with FXS +Aut and may represent strengths unique to this variant of autism. ${ }^{11}$ Overall patterns of social behavior between FXS+Aut and iAut were most similar for social initiation items (Figure 4). Between-group differences in repetitive and social behaviors held-up to post-hoc analyses using matched samples and conservative tests of significance.

These findings are consistent with existing work concerning the behavioral phenotype of FXS + Aut and further inform the process by which autism comes to be diagnosed in children with FXS. As with previous research, we found that children with FXS who meet diagnostic criteria for autism may do so due to hyper-expression of the FXS behavioral phenotype (e.g. excessive social avoidance, elevated repetitive motor behavior) rather than a distinct condition that sets them apart from children with FXS without autism. ${ }^{6}, 11,22,24$ That autistic behaviors associated with FXS occur on a continuum mirrors the forthcoming collapse of diagnostic categories into a single classification of autism spectrum disorder in $D S M-5$, an acknowledgement, in part, of the dimensional nature of autism. ${ }^{39}$ Attention to the dimensional expression of autistic behaviors among individual children with FXS may be more meaningful than attempting to impose categorical distinctions which imply a brightline difference. ${ }^{6,11,21}$ Repetitive behaviors and social deficits associated with the FXS phenotype occur on a continuum, and children meeting criteria for autism because of behaviors constituting these domains may not be qualitatively different from those who do not. ${ }^{7}, 19,40$ Although we did not include boys with FXS (w/o aut) in our analyses, radar graphs of behavior patterns in all three groups lend support to a dimensional view of autistic behavior associated with FXS (Figure 4). Interestingly, there is evidence that this continuum of autistic behavior extends beyond genetically defined categorical boundaries to unaffected first degree relatives of individuals with FXS and iAut. ${ }^{41}$

The present findings also suggest that children with FXS + Aut may be more attuned to social cues than their counterparts with iAut, capable of responding with appropriate social modalities when pressed to do so. ${ }^{18,42}$ For iAut, social deficits likely stem from a failure to attend to social information and general social indifference, thus precluding appropriate social behavior. ${ }^{43,}{ }^{44}$ However, a lack of pro-social initiative alone does not necessarily imply the absence of social ability or social awareness. For those with FXS + Aut, social initiation deficits may reflect the social anxiety common to children with FXS. ${ }^{45}, 46$ Although children with FXS + Aut may avoid making social bids (e.g. Showing, Requesting, IJA), they may be capable of appropriately responding to social bids made by others (e.g. Responsive Social Smiling, Facial Expressions, RJA) despite rather severe cognitive impairment. Social initiation deficits related to social anxiety may be particularly pronounced in assessment settings with an unfamiliar assessor, contributing to the 
perception of general social impairment. Previous work has demonstrated that children with FXS in general show a decrease in socially avoidant behavior over time in the presence of new people, with children with FXS + Aut particularly slow to "warm-up" to unfamiliar persons. $^{20}$

A clear benefit of the homogeneity of FXS is that it provides an ideal model for identifying neurobiological mechanisms associated with a known variant of autism. ${ }^{47-49}$ Recent work has demonstrated that the neuroanatomical signature of FXS + Aut differs from iAut, with the former characterized by highly enlarged caudate and small amygdale and the latter characterized by modest caudate and amygdale enlargement. ${ }^{28,29,50}$ Complex forms of repetitive behavior, such as compulsive and ritualistic behavior, have been positively associated with caudate volume among individuals with iAut. ${ }^{51}$ Given that caudate in FXS is enlarged relative to iAut, it is surprising that our results indicate that compulsive and ritual behaviors are significantly lower among boys with FXS + Aut. This may suggest mediation by associated structures or altered striatal connectivity, or perhaps an altogether different neural basis. ${ }^{28}$ Separate lines of research have found that social avoidance is associated with neuroendocrine dysfunction in children with FXS, ${ }^{21,52}$ while social indifference or inattention to social cues has been tied to amygdale overgrowth and dysfunction in iAut. ${ }^{53,54}$ The neurodevelopmental pathways leading to seemingly similar, but qualitatively different, social deficits between iAut and FXS + Aut may themselves differ with regard to both brain (amygdale volume) and behavior (social indifference versus social avoidance). Direct investigation of such specific brain-behavior relationships is needed, and FXS affords the opportunity to do so absent the 'noise' characteristic of idiopathic autism.

There are a number of limitations to the present study. The use of item-level ADOS items to capture and compare social behaviors represents unconventional use of this measure. We used ADOS social items as discrete and standardized presses for specific social behaviors. Though reliability is generally strong at the item-level, and severity scores generated to control for variance, rater error and individual differences may be pronounced at this level of analysis. Our delineation between items primarily concerning social initiation and social response was made a priori based on item descriptions and experience with the ADOS. However, this qualitative approach has not been psychometrically validated, and the extent to which a behavior such as socially directed facial expressions, for instance, may be considered as social response rather than initiation is certainly open to debate. It is feasible that scores on ADOS social items reflect some degree of both social initiation and social response contexts. Because the ADOS served both to behaviorally define autism and assess social behavior related to autism, it is possible that group differences in social behavior result more from means of sample selection. As with previous work of this type, there is the possibility that the present findings are impacted by ascertainment bias inherent to comparing individuals with a genetically defined disorder to clinically referred individuals. For convergent validity, future work should consider employing a variety of measures to better characterize behaviors of interest. The present study focused on boys meeting criteria for autistic disorder; future work might extend analyses to females and/or autism spectrum disorder.

The present study revealed distinct patterns of repetitive and social behavior among young boys with FXS + Aut compared to iAut. In our sample, FXS + Aut was characterized by elevated repetitive motor behaviors and social avoidance (impaired pro-social behavior), but fewer compulsive and ritual behaviors and some evidence of less impaired social responsiveness than children with iAut. Patterns of repetitive and social behavior in FXS + Aut appear to resemble the phenotype of FXS generally, though more pronounced, and are in part discordant with classic autistic disorder given the relative absence of a number of cardinal features (e.g. compulsivity, impaired social smile). One advantage of a dimensional 
rather than categorical approach to behavior is the ability to detect subtle but potentially meaningful differences between individuals and groups. When William Nyhan coined the term "behavioral phenotype", he proposed that cataloguing specific repertoires of behavior would serve to differentiate syndromes which seemingly overlap. ${ }^{55}$ As we continue to catalogue specific behavioral profiles, such as that for FXS + Aut, so do we provide important insights into the pathogenesis of defining behavioral features associated with neurodevelopmental disorders.

\section{Acknowledgments}

This research was supported by the National Institutes of Health grants MH64708-05 (A.L.R., J.P.), MH61696 (J.P.), MH050047 (A.L.R.), and P30 HD03110 (J.P.).

The authors sincerely thank all the families who participated in this project.

\section{References}

1. Crawford DC, Acuña JM, Sherman SL. FMR1 and the fragile X syndrome: human genome epidemiology review. Genet Med. 2001; 3:359-371. [PubMed: 11545690]

2. Boyle L, Kaufman WE. The behavioral phenotype of FMR1 mutations. Am J Med Genet C Semin Med Genet. 2010; 154C:469-476. [PubMed: 20981777]

3. Feinstein C, Reiss AL. Autism: the point of view from fragile X studies. J Autism Dev Disord. 1998; 28:393-405. [PubMed: 9813775]

4. Fisch GS. What is associated with the fragile X syndrome? Am J Med Genet A. 1993; 48:112-121.

5. Harris SW, Hessl D, Goodlin-Jones B, et al. Autism profiles of males with fragile X syndrome. Am J Ment Retard. 2008; 113:427-438. [PubMed: 19127654]

6. Hall SS, Lightbody AA, Hirt M, Rezvani A, Reiss AL. Autism in fragile X syndrome: a category mistake? J Am Acad Child Adolesc Psychiatry. 2010; 49:921-933. [PubMed: 20732628]

7. Rogers SJ, Wehner DE, Hagerman R. The behavioral phenotype in fragile X: symptoms of autism in very young children with fragile $\mathrm{X}$ syndrome, idiopathic autism, and other developmental disorders. J Dev Behav Pediatr. 2001; 22:409-417. [PubMed: 11773805]

8. Geschwind D, Levitt P. Autism spectrum disorders: developmental disconnection syndromes. Curr Opin Neurobiol. 2007; 17:103-111. [PubMed: 17275283]

9. Hagerman R, Hoem G, Hagerman P. Fragile X and autism: intertwined at the molecular level leading to targeted treatments. Mol Autism. 2010; 1:12. [PubMed: 20858229]

10. Baranek GT, Danko CD, Skinner ML, et al. Video analysis of sensory-motor features in infants with fragile X syndrome at 9-12 months of age. J Autism Dev Disord. 2005; 35:645-656. [PubMed: 16172809]

11. Kau ASM, Tierney E, Bukelis I, et al. Social behavior profile in young males with fragile $X$ syndrome: characteristics and specificity. Am J Med Genet A. 2004; 126:9-17. [PubMed: 15039968]

12. Symons FJ, Byiers BJ, Raspa M, Bishop E, Bailey DB. Self-injurious behavior and fragile X syndrome: findings from the national fragile X survey. Am J Intellect Dev Disabil. 2010; 115:473481. [PubMed: 20946000]

13. Brock M, Hatton D. Distinguishing features of autism in boys with fragile X syndrome. J Intellect Disabil Res. 2010; 54:894-905. [PubMed: 20704635]

14. McDuffie A, Abbeduto L, Lewis P, et al. Autism spectrum disorder in children and adolescents with fragile $\mathrm{X}$ syndrome: within-syndrome differences and age-related changes. Am J Intellect Dev Disabil. 2010; 115:307-326. [PubMed: 20567604]

15. Morgan L, Wetherby AM, Barber A. Repetitive and stereotyped movements in children with autism spectrum disorders late in the second year of life. J Child Psychol Psychiatry. 2008; 49:826-837. [PubMed: 18503532] 
16. Hall SS, Lightbody AA, Reiss AL. Compulsive, self-injurious, and autistic behavior in children and adolescents with fragile X syndrome. Am J Ment Retard. 2008; 113:44-53. [PubMed: 18173299]

17. Symons FJ, Clark RD, Hatton DD, Skinner M, Bailey DB. Self-injurious behavior in young boys with fragile X syndrome. Am J Med Genet A. 2003; 118A:115-121. [PubMed: 12655491]

18. Budimirovic DB, Bukelis I, Cox C, Gray RM, Tierney E, Kaufmann WE. Autism spectrum disorder in fragile $\mathrm{X}$ syndrome: differential contribution of adaptive socialization and social withdrawal. Am J Med Genet A. 2006; 140A:1814-1826. [PubMed: 16906564]

19. Kaufmann WE, Cortell R, Kau ASM, et al. Autism spectrum disorder in fragile X syndrome: communication, social interaction, and specific behaviors. Am J Med Genet A. 2004; 129:225234. [PubMed: 15326621]

20. Roberts JE, Weisenfeld LAH, Hatton DD, Heath M, Kaufmann WE. Social approach and autistic behavior in children with fragile X syndrome. J Autism Dev Disord. 2007; 37:1748-1760. [PubMed: 17180715]

21. Hall S, DeBernardis M, Reiss A. Social escape behaviors in children with fragile X syndrome. J Autism Dev Disord. 2006; 36:935-947. [PubMed: 16897394]

22. Bailey DB, Mesibov GB, Hatton DD, Clark RD, Roberts JE, Mayhew L. Autistic behavior in young boys with fragile X syndrome. J Autism Dev Disord. 1998; 28:499-508. [PubMed: 9932236]

23. Dissanayake C, Bui Q, Bulhak-Paterson D, Huggins R, Loesch DZ. Behavioural and cognitive phenotypes in idiopathic autism versus autism associated with fragile X syndrome. J Child Psychol Psychiatry. 2009; 50:290-299. [PubMed: 19175809]

24. Smith LE, Barker ET, Seltzer MM, Abbeduto L, Greenberg JS. Behavioral phenotype of fragile X syndrome in adolescence and adulthood. Am J Intellect Dev Disabil. 2012; 117:1-17. [PubMed: 22264109]

25. Hagerman RJ, Rivera SM, Hagerman PJ. The fragile X family of disorders: a model for autism and targeted treatments. Curr Pediatr Rev. 2008; 4:40-52.

26. Steinhausen H, von Gontard A, Spohr H, et al. Behavioral phenotypes in four mental retardation syndromes: fetal alcohol syndrome, Prader-Willi syndrome, fragile X syndrome, and tuberosis sclerosis. Am J Med Genet A. 2002; 111:381-387.

27. Esbensen AJ, Seltzer MM, Lam KS, Bodfish JW. Age-related differences in restricted repetitive behaviors in autism spectrum disorders. J Autism Dev Disord. 2009; 39:57-66. [PubMed: 18566881]

28. Hazlett HC, Poe MD, Lightbody AA, et al. Teasing apart the heterogeneity of autism: same behavior, different brains in toddlers with fragile X syndrome and autism. J Neurodev Disord. 2009; 1:81-90. [PubMed: 20700390]

29. Hoeft F, Carter JC, Lightbody AA, Hazlett HC, Piven J, Reiss AL. Region-specific alterations in brain development in one- to three-year-old boys with fragile X syndrome. Proc Natl Acad Sci U S A. 2010; 107:9335-9339. [PubMed: 20439717]

30. Bodfish JW, Symons FJ, Parker DE, Lewis MH. Varieties of repetitive behavior in autism: Comparisons to mental retardation. J Autism Dev Disord. 2000; 30:237-243. [PubMed: 11055459]

31. Lord, C.; Rutter, M.; DiLavore, PC.; Risi, S. Autism Diagnostic Observation Schedule. Los Angeles, CA: Western Psychological Services; 2002.

32. Lord C, Rutter M, LeCouteur A. Autism Diagnostic Interview-Revised: a revised version of a diagnostic interview for caregivers of individuals with possible pervasive developmental disorders. J Autism Dev Disord. 1994; 24:659-685. [PubMed: 7814313]

33. Willemsen R, Mohkamsing S, de Vries B, et al. Rapid antibody test for fragile X syndrome. Lancet. 1995; 345:1147-1148. [PubMed: 7723547]

34. Mirenda P, Smith IM, Vailancourt T, et al. Validating the Repetitive Behavior Scale-revised in young children with autism spectrum disorders. J Autism Dev Disord. 2009; 40:1521-1530. [PubMed: 20405194]

35. Gotham K, Pickles A, Lord C. Standardizing ADOS scores for a measure of severity in autism spectrum disorders. J Autism Dev Disord. 2009; 39:693-705. [PubMed: 19082876] 
36. Mullen, EM. Mullen Scales of Early Learning: AGS edition. Circle Pines, MN: AGS Publishing; 1995.

37. Carcani-Rathwell I, Rabe-Hasketh S, Santosh PJ. Repetitive and stereotyped behaviours in pervasive developmental disorders. J Child Psychol Psychiatry. 2006; 47:573-581. [PubMed: 16712634]

38. Hus V, Pickles A, Cook EH Jr, Risi S, Lord C. Using the Autism Diagnostic Interview-Revised to increase phenotypic homogeneity in genetic studies of autism. Biol Psychiatry. 2007; 61:438-448. [PubMed: 17276746]

39. Lord C, Jones RM. Annual research review: rethinking the classification of autism spectrum disorders. J Child Psychol Psychiatry. 2012; 53:490-509. [PubMed: 22486486]

40. Moss J, Oliver C, Arron K, Burbidge C, Berg K. The prevalence and phenomenology of repetitive behavior in genetic syndromes. J Autism Dev Disord. 2009; 39:572-588. [PubMed: 19037716]

41. Losh M, Klusek J, Martin GE, Sideris J, Parlier M, Piven J. Defining genetically meaningful language and personality traits in relatives of individuals with fragile $\mathrm{X}$ syndrome and relatives of individuals with autism. Am J Med Genet B Neuropsychiatr Genet. 2012; 159B(6):660-668. [PubMed: 22693142]

42. Ferrier LJ, Bashir AS, Meryash DL, Johnston J, Wolff P. Conversations skills of individuals with fragile X syndrome: a comparison with autism and Down syndrome. Dev Med Child Neurol. 1991; 33:776-788. [PubMed: 1834506]

43. Dawson G, Toth K, Abbott R, et al. Early social attention impairments in autism: social orienting, joint attention, and attention to distress. Dev Psychol. 2004; 40:271-283. [PubMed: 14979766]

44. Steer CD, Golding J, Bolton PF. Traits contributing to the autism spectrum. PLoS One. 2010; 5(9):e12633. [PubMed: 20838614]

45. Adrien JL, Barthélemy A, Perrot A, et al. Validity and reliability of the Infant Behavioral Summarized Evaluation (IBSE): a rating scale for the assessment of young children with autism and developmental disorders. J Autism Dev Disord. 1992; 22:375-394. [PubMed: 1383188]

46. Bailey DB, Nelson D. The nature and consequences of fragile X syndrome. Ment Retard Dev Disabil Res Rev. 1995; 1:238-244.

47. Abrahams BS, Geschwind DH. Advances in autism genetics: on the threshold of a new neurobiology. Nat Rev Genet. 2008; 9:341-355. [PubMed: 18414403]

48. Belmonte MK, Bourgeron T. Fragile $\mathrm{x}$ syndrome and autism at the intersection of genetic and neural networks. Nat Neurosci. 2006; 9:1221-1225. [PubMed: 17001341]

49. Moy SS, Nadler JJ, Young NB, et al. Social approach in genetically engineered mouse lines relevant to autism. Genes Brain Behav. 2009; 8:129-142. [PubMed: 19016890]

50. Hoeft F, Walter E, Lightbody AA, et al. Neuroanatomical differences in toddler boys with fragile X syndrome and idiopathic autism. Arch Gen Psychiatry. 2001; 68:295-305. [PubMed: 21041609]

51. Hollander E, Anagnostou E, Chaplin W, et al. Striatal volume on magnetic resonance imaging and repetitive behaviors in autism. Biol Psychiatry. 2005; 58:226-232. [PubMed: 15939406]

52. Roberts JE, Clarke MA, Alcorn K, Carter JC, Long ACJ. Autistic behavior in boys with fragile X syndrome: social approach and HPA-axis dysfunction. J Neurodev Disord. 2009; 1:283-291. [PubMed: 21547720]

53. Munson J, Dawson G, Abbott R, et al. Amygdalar volume and behavioral development in autism. Arch Gen Psychiatry. 2006; 63:686-693. [PubMed: 16754842]

54. Mosconi MW, Cody-Hazlett H, Poe MD, Gerig G, Gimpel-Smith R, Piven J. Longitudinal study of amygdala volume and joint attention in 2- to 4-year old children with autism. Arch Gen Psychiatry. 2009; 66:509-516. [PubMed: 19414710]

55. Nyhan WL. Behavioral phenotypes in organic genetic disease: presidential address to the Society for Pediatric Research, May 1, 1971. Pediatr Res. 1972; 6:1-9. [PubMed: 5046967] 


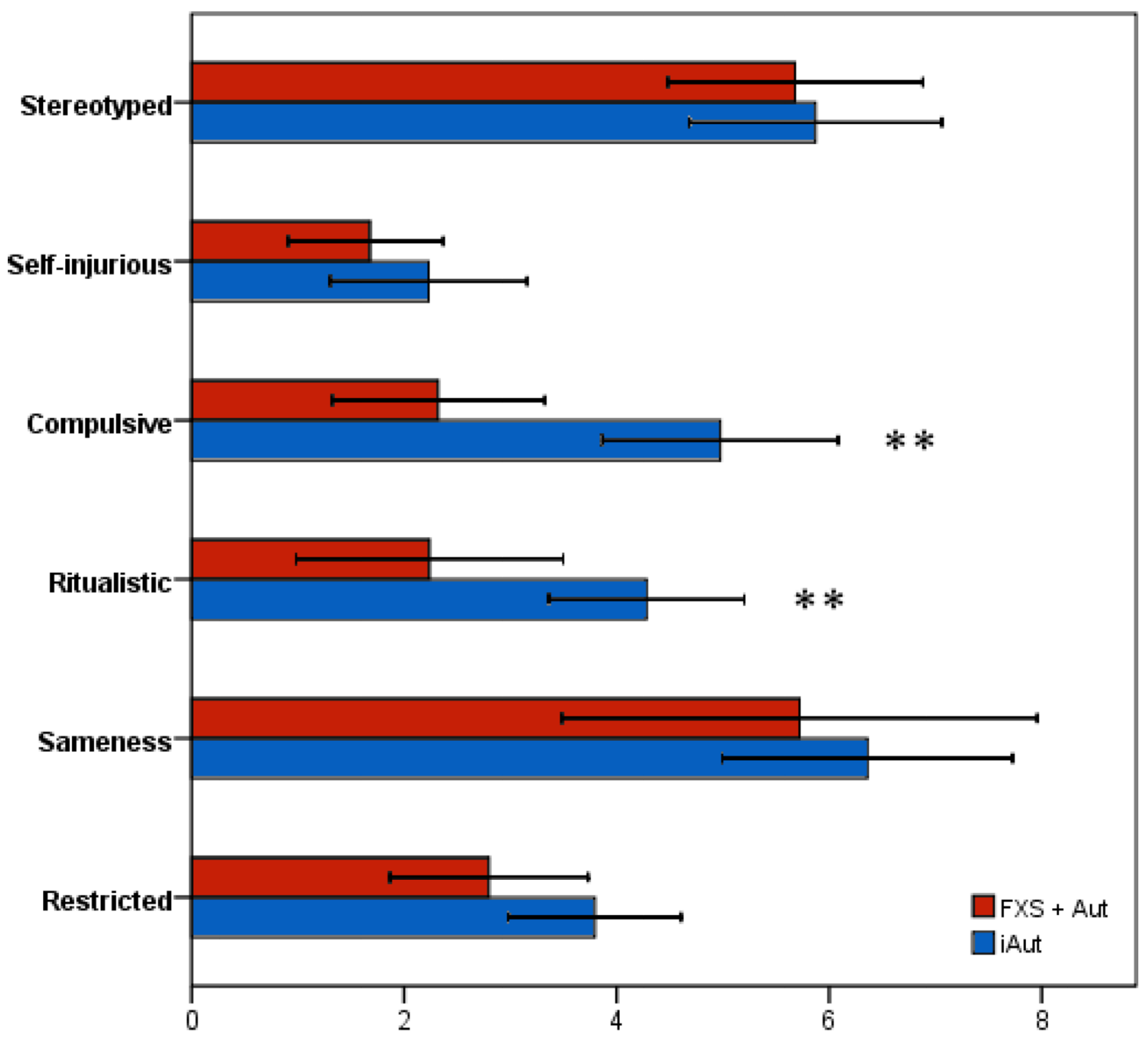

Mean RBS-R Subscale Score

Figure 1.

Mean and 95\% confidence intervals for Repetitive Behavior Scale, Revised (RBS-R) subscale scores for boys with fragile X syndrome and autism (FXS + Aut) and idiopathic autism (iAut). Note: $* p<.05, * * p<.01$, *** $p<.001$. 


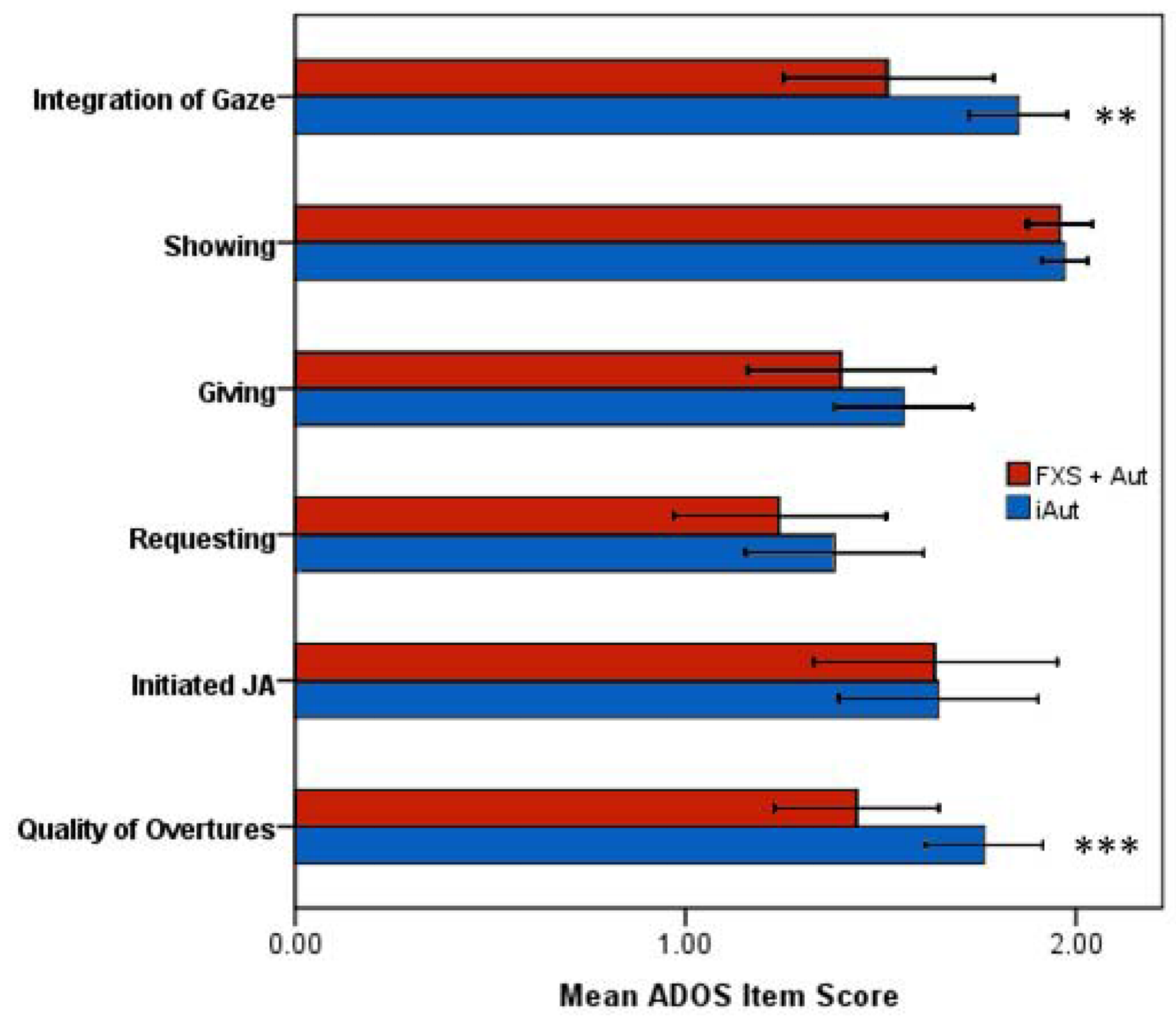

Figure 2.

Mean and 95\% confidence intervals Autism Diagnostic Observation Schedule (ADOS) social domain scores (social initiation) for boys with fragile X syndrome and autism (FXS + Aut) and idiopathic autism (iAut). Note: JA = Joint Attention; * $p<.05, * * p<.01, * * * p$ $<.001$. 


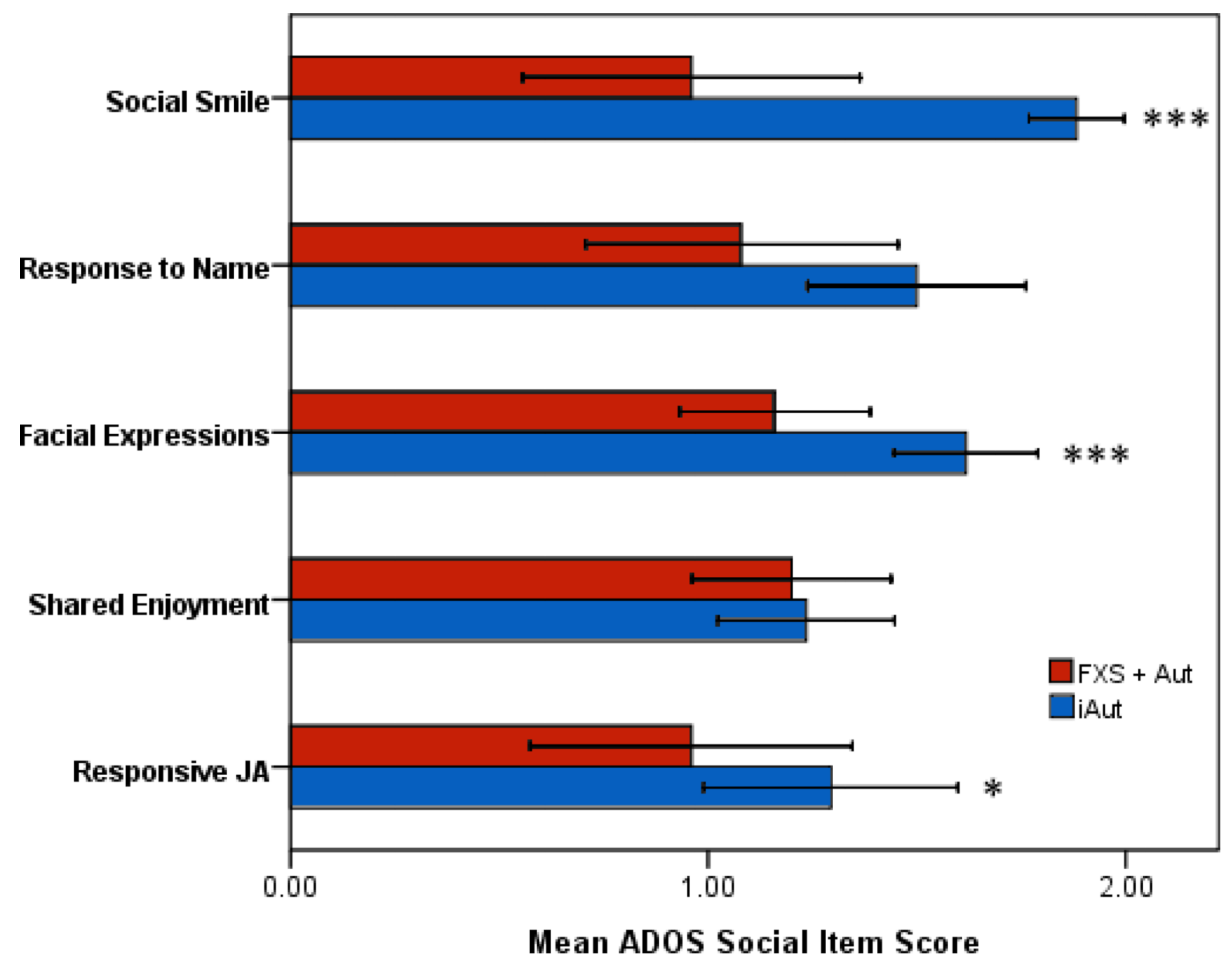

Figure 3.

Mean and 95\% confidence intervals for Autism Diagnostic Observation Schedule (ADOS) social domain scores (social response) for boys with fragile X syndrome and autism (FXS + Aut) and idiopathic autism (iAut). Note: JA $=$ Joint Attention; $* p<.05, * * p<.01,{ }^{* * *} p$ $<.001$. 


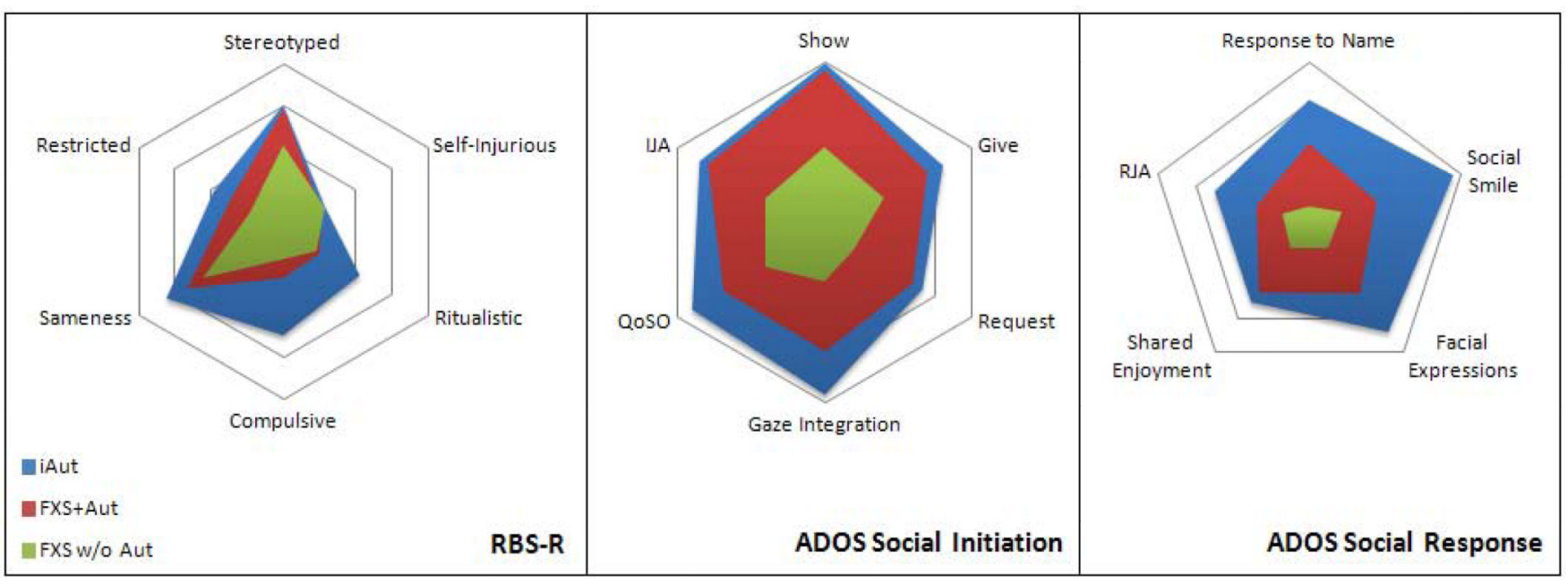

Figure 4.

Radar graphs of Repetitive Behavior Scale, Revised (RBS-R) subscales and Autism Diagnostic Observation Schedule (ADOS) social initiation and social response items for boys with fragile X syndrome and autism (FXS + Aut), fragile X without autism (FXS w/o Aut), and idiopathic autism (iAut). Note: IJA = Initiated Joint Attention; QoSO = Quality of Social Overtures; RJA = Response to Joint Attention. 


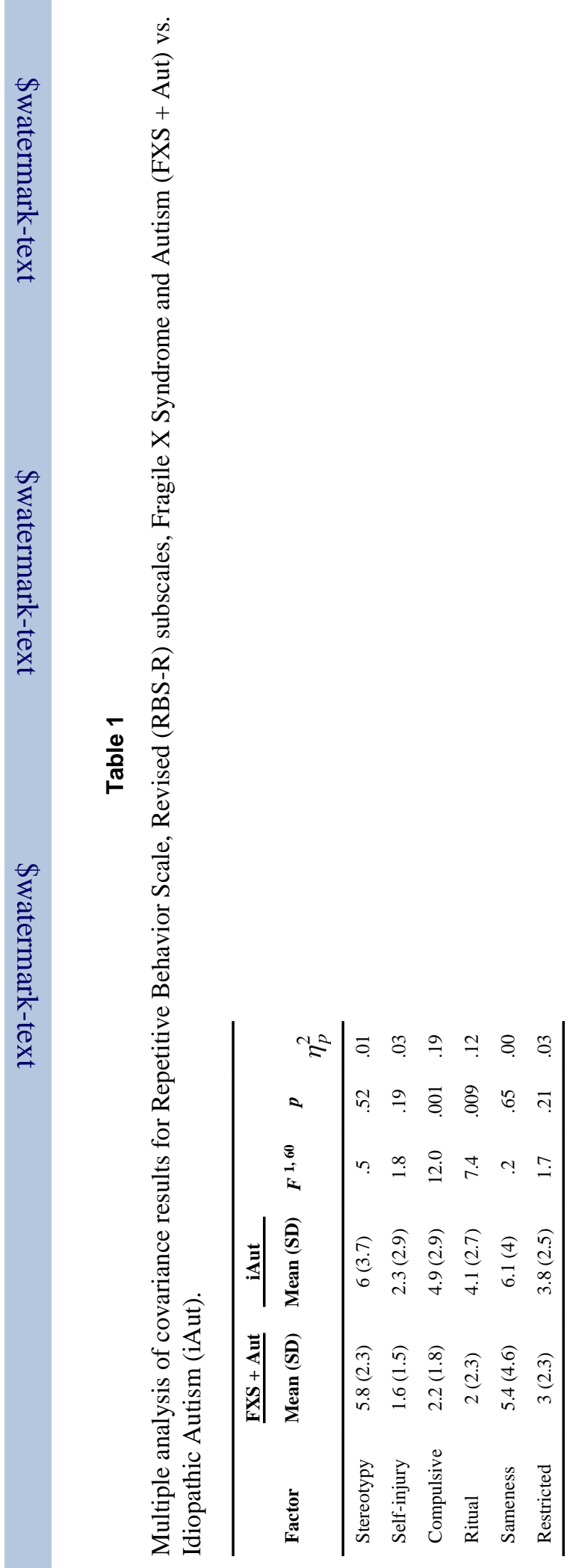




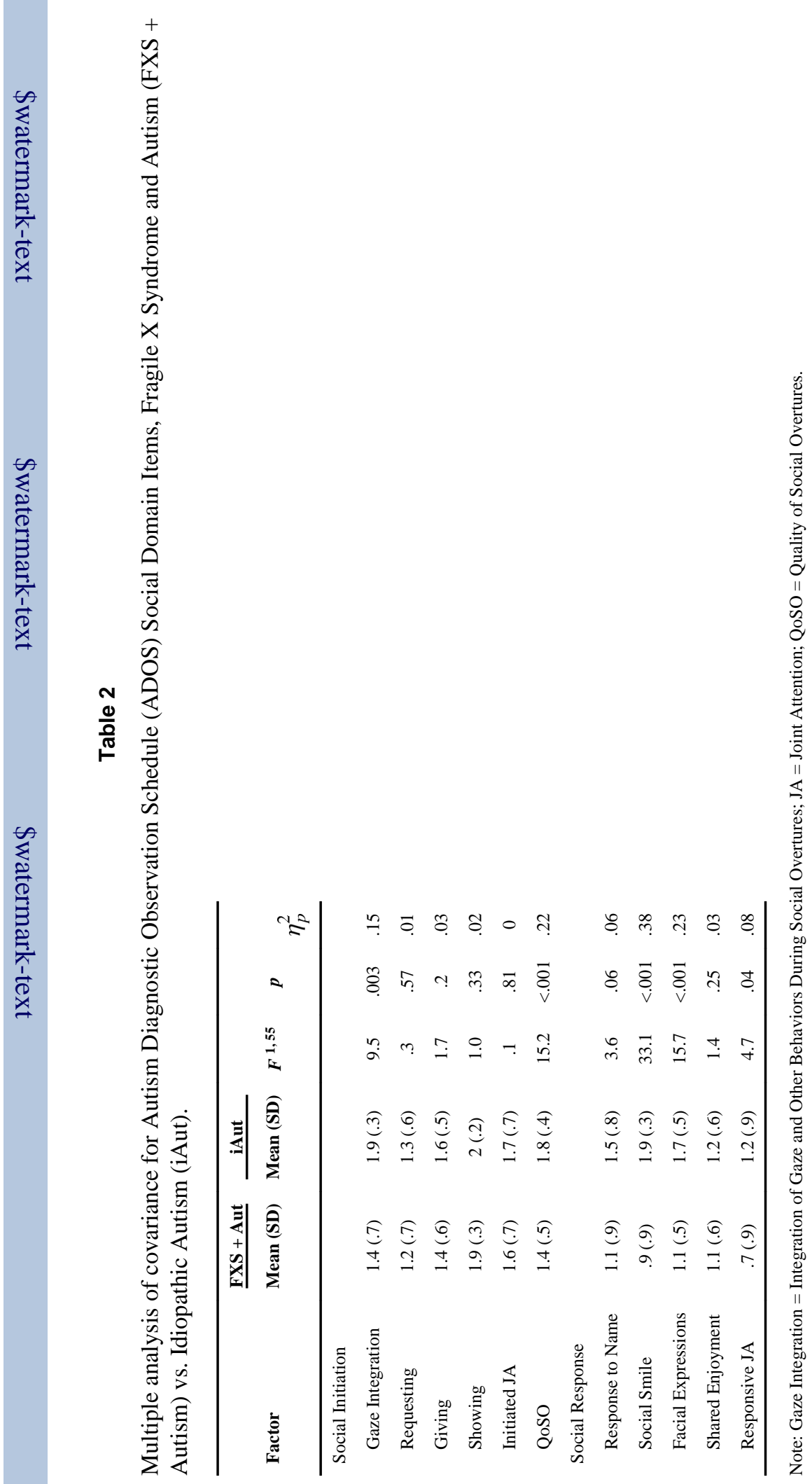

J Am Acad Child Adolesc Psychiatry. Author manuscript; available in PMC 2013 December 01. 